\title{
Accounting for Immigrant Status when Calculating Cancer Incidence Rates for Bangkok
}

\author{
Rangsiya Buasom ${ }^{1}$, David Roder ${ }^{2}$, Nisit Singhakosit ${ }^{1}$, Siriphon Sitthikong ${ }^{1}$, \\ Elizabeth Buckley², Suleeporn Sangrajrang**
}

\begin{abstract}
Objective: Cancer-registry data are crucial for definingcancer incidence rates for use in setting service priorities and monitoringservice effects. This applies in Thailand where cancer is the leading cause of death and service needs are high. The Bangkok Cancer Registry (population-based) was established in 1990to determine cancer incidence rates for Bangkok. This proved difficult, however, because the Bangkokpopulation ( $>8$ million) fluctuates with numbers of temporary visitors, many of whom visit Bangkok temporarily for services. If these visitors are mis-categorized as usual residents, cancer incidence rateswould be inflated. During 2013-2015, residential addresses on the Registry were cross-checked against official addresses on the National Civil Registration records of the Ministry of Interior. The effectsof this cross-checking on incidence rates are discussed. Methods: Residential addresses recorded on the Registry for cancer diagnoses in 2013-2015 were corrected using official Ministry data. Effects on numbers of recorded cancers and crude and directly age-standardized rates (World Population) were determined. Results: Of 44,813 cancer casesdiagnosed and recorded on the Registryduring 2013-2015, 36,327 (81.1\%) had an official Bangkok address. When limiting analyses to these cases, the crude incidencefor all cancer sites combined reduced by $18.9 \%$ (19.7\% for males and $18.3 \%$ for females). Corresponding reductions in age-standardized incidence rates were $20.0 \%$ for males and $18.8 \%$ for females. These reductions varied for common cancer sites:in males, from $14.8 \%$ for lung to $25.9 \%$ for colorectal cancer; and in females, from $12.9 \%$ for lung to $24.0 \%$ for cervical cancer. Conclusions: These differences are considered sufficient in magnitude to justifyroutine use of official residential data when calculating cancer incidence rates for Bangkok. If these rates are to be compared with comparable rates for other Asian citiesthat serve broader populations, equivalent methodologies for determining residential status would be needed for all cities.
\end{abstract}

Keywords: Cancer incidence- Bangkok- accuracy

Asian Pac J Cancer Prev, 20 (3), 737-741

\section{Introduction}

Bangkok, the capital of Thailand,is located centrallyon the banks of Chao Praya River.The city extends over 1,569 square kilometres and has a population that exceeds 8 million (i.e., $12.6 \%$ of the Thai population) (NESDB, 2017). It is bordered by Nonthaburi and Pathumthani provinces to the North, Chachoengsoa to the East, Samut Prakan to the South, and Nakhon Pathom and Samut Sakhon to the West. Over 14 million people ( $22.2 \%$ of the national population) were recorded to live in the broader Bangkok Metropolitan Region in the 2010 census. This far surpasses the population sizesof other urban centres in Thailand.

The Bangkok Cancer Registry (the Registry) was established in 1990 at the NationalCancer Institute of Thailand (NCI) with technical support from the International
Agency for Research on Cancer for population-based monitoring (Vatanasapt et al., 1993; (Martin et al, 2001). Initially, CanReg software from the International Agency for Research on Cancer (IARC) was used to create and manage the database (Vatanasapt et al., 1993). In 2012, NCI developed a customized program called the "Thai Cancer Based", which was further developed in 2015 as a web-based system. Currently, the Registry has obtained voluntary cooperation from local hospitals, including small private hospitals to register cases. The total participation is around 500 hospitals across Thailand which provide cancer data to through the one "Thai Cancer Based" system. This helps to avoid duplicative registrations, improves data quality, and will facilitate planned extensions of the Registry to become a national population-based registry.

Current registrations for Bangkok residents cover

${ }^{1}$ Cancer Registry Unit, ${ }^{3}$ Research Division, National Cancer Institute, Ratchathewi Road, Bangkok, Thailand, ${ }^{2}$ Cancer Epidemiology and Population Health, Centre for Population Health Research, University of South Australia, Australia. *For Correspondence: sulee@health.moph.go.th 
122 hospitals comprising four university hospitals, 33 government hospitals and 85 private hospitals. Estimatingfrom hospital coverage, Registry data would cover $95 \%$ or more of the cancers diagnosed in Bangkok residents.

A major challenge when determining cancer rates for Bangkok,is accounting for effects of population inflows for care from surrounding areas. The Thai National Civil Registration system can now be used to determine residential status (NationalIdentity Card Act. B.E.2505). This systemhas evolved significantly over the decades from a manual, paper-based system to acentralized and online electronic system, providing better access to accurate records of official places of residence.

In the past, patients could receive medical services in hospitalswithout presenting ID cards that identified their official place of residence. Lack of reliable evidence of residential addresses was a problem since self-reported addresses were known to be unreliable and to sometimes include temporary Bangkok addresses for short-stays, asundertaken for access to clinical services.

Thailand has universal health coverage, with three major national health insurance schemes, i.e., the Universal Health Coverage Scheme (covering 48 million); Social Health Insurance (covering 10.6 million) and the Civil Servant Medical Benefit Scheme (covering 4.4 million) (Tangcharoensathien V, et al., 2018), which include coverage for cancer treatment. Since Thailand's Universal Coverage Scheme was implemented in all Thai provincesin January 2002, patients have been asked to show their ID cardsto gain reimbursementfor clinical charges (NHSO, 2012). In addition, with the introduction of digital technology,official addresses of patients can now be checked and any missing fields are filled using ID numbers from the national civil registration files of the Ministry of Interior. This enables exclusion of data for cancer patients whose officialaddresses are outside Bangkok, thus reducing and better defining cancer incidence rates for the officialresidential population of Bangkok.

This paperreports the effect of using official addresses on incidence rates by primary cancer site.

\section{Materials and Methods}

\section{Data Collection}

Projection population data was sourced from the Office of the National Economic and Social Development Board (NESDB) for each province in Thailand, totaling 23,935,307 people during 2013-15 (11,513,528 male and 12,421,779 female). Data on cancer incidence cases were collected from hospitals, pathology and haematology laboratories, and death certificates during 2013-2015. Data items includednames, official civil registration ID number, Registry number, residential address, date of birth, age at diagnosis, sex, dates and methods of diagnosis, topographic site of the cancer, histology type, extent of cancer spread, and vital status. Operational details have been reported in greater detail previously (Imsamran et al., 2015).

The International Classification of Diseases for
Oncology Third Edition (ICD-O 3) is used to code cancers by primary siteand morphology type (WHO, 2000). The data are recorded on the Registry using the Thai Cancer Based (TCB) Software (NCI, 2017).

Because Thai people often believe that they will obtain better treatment in Bangkok than in regional settings, Bangkok hospitals generally have patients from other provinces. While self-reported residential addresses have been recorded by hospitals and laboratories since the Registry inception, partlyto enable follow-up, these addresses often have includedBangkok addresses used only for temporary stays.

For the first time in 2015,official residential addresses held by Ministry of Interior became available for cross-checking with Registry recorded addresses and for filling missing fields. For the patients found through Ministry records to have an official address in Bangkok, the numbers of years lived in Bangkok were also obtained from Ministry records. For patients with anofficialaddress in other provinces, checks could be made whether they had previously lived in Bangkok.

\section{Identifying duplicative data in other registries}

Currently, there are 500 hospitals in Thailand which recordcancersfor the national TCBregistry program. All cases recorded in the Bangkok Cancer Registry were checked to determine whether there were duplicate entries with other Thai hospitals and registries.

\section{Data analyses}

Numbers of cancers, crude and directly age-standardized (World Population) incidence rates were calculated, using all cancers registered on the Registry for: (a) all invasive cancers collectively; and (b) common primary sites (MacLennan, 1991). This was done initially for all notifications on the Registry, irrespective of official residential address, and then only for patients who had an official address in Bangkok at time of diagnosis, in order to quantify differences.

\section{Results}

\section{All registered cancers}

A totalof 44,813 newly diagnosed cancer cases were registered by the Registry in 2013-2015, 24,839 $(55.4 \%)$ in females and 19,974 (44.6\%) in males. Their age distribution in years was: $<40=9.3 \% ; 40-49=13.8 \%$; $50-59=25.1 \%$; $60-69=25.1 \%$; and $70+=26.7 \%$.

Leading primary sites for both sexes combined included the breast $(7,483,16.7 \%)$, colon/rectum $(5,911,13.2 \%)$, and lung $(4,879,10.9 \%)$, and liver $(4,148$, 9.3\%)whereas 2,165 (4.8\%)had cervical cancer (Table 1). For all sites combined, the age-standardized incidence was $4.1 \%$ higher for males than females. Predictably, virtually all breast cancers affected females (99.2\%). By comparison, males had a higher age-standardized incidence than females for cancers of the liver $(+194.8 \%)$, lung $(+109.5 \%)$, and colon/rectum $(+61.3 \%)$.

In general, data quality appeared to be higher for females than males as indicated by the $\%$ death certificate only (DCO) 
Table 1. Crude and Age-standardized Rates Per 100,000 for All Registered Cases and Those Confirmed to have a Permanent Address in Bangkok; Bangkok Cancer Registry, 2013-2015

\begin{tabular}{|c|c|c|c|c|c|c|c|c|c|c|c|c|}
\hline \multirow[t]{3}{*}{ Cancer } & \multicolumn{6}{|c|}{ All registered } & \multicolumn{6}{|c|}{ Cases with a confirmed Bangkok address } \\
\hline & \multicolumn{2}{|c|}{ Number } & \multicolumn{2}{|c|}{ Crude rate } & \multicolumn{2}{|c|}{$\mathrm{ASR}^{*}$} & \multicolumn{2}{|c|}{ Number } & \multicolumn{2}{|c|}{ Crude rate } & \multicolumn{2}{|c|}{$\mathrm{ASR}^{*}$} \\
\hline & Male & Female & Male & Female & Male & Female & Male & Female & Male & Female & Male & Female \\
\hline Colorectal & 3,288 & 2,623 & 28.6 & 21.2 & 26.3 & 16.3 & 2,443 & 2,027 & 21.2 & 16.4 & 19.5 & 12.5 \\
\hline Liver & 2,917 & 1,231 & 25.3 & 9.9 & 22.7 & 7.7 & 2,440 & 1,052 & 21.2 & 8.5 & 18.9 & 6.5 \\
\hline Lung & 3,007 & 1,872 & 26.1 & 15.1 & 24.3 & 11.6 & 2,568 & 1,625 & 22.3 & 13.1 & 20.7 & 10.1 \\
\hline Breast & 60 & 7,423 & 0.5 & 59.8 & 0.5 & 44.6 & 45 & 6,047 & 0.4 & 48.7 & 0.3 & 36.2 \\
\hline Cervix & - & 2,165 & - & 17.4 & - & 12.9 & - & 1,641 & - & 13.2 & 0.0 & 9.8 \\
\hline Other sites & 10,702 & 9,525 & 92.9 & 76.5 & 87.5 & 61.9 & 8,548 & 7,891 & 74.2 & 63.4 & 69.7 & 50.7 \\
\hline All sites & 19,974 & 24,839 & 173.4 & 199.9 & 161.3 & 155.0 & 16,044 & 20,283 & 139.3 & 163.3 & 129.1 & 125.8 \\
\hline
\end{tabular}

* ASR-age-standardized rate (World Population)

Table 2. For All Registered Cases, the Percentage Morphologically Verified and the Percentage Notified from Death Certificate Evidence Only; Bangkok Cancer Registry, 2013-2015*

\begin{tabular}{lcccccc}
\hline Cancer & \multicolumn{2}{c}{ Number } & \multicolumn{2}{c}{ All registered } \\
& Male & Female & \% MV & \%DCO & \% MV & \% DCO \\
\hline Colorectal & 3,288 & 2,623 & 84.5 & 0.7 & 82.5 & 0.4 \\
Liver & 2,917 & 1,231 & 38.5 & 13.5 & 42.6 & 17.2 \\
Lung & 3,007 & 1,872 & 68.4 & 12.8 & 68.4 & 14.9 \\
Breast & 60 & 7,423 & 100.0 & 2.9 & 86.7 & 0.7 \\
Cervix & - & 2,165 & - & - & 84.5 & 1.5 \\
Other sites & 10,702 & 9,525 & 82.4 & 4.2 & 83.7 & 4.8 \\
All sites & 19,974 & 24,839 & 75.9 & 6.0 & 81.7 & 3.7 \\
\hline
\end{tabular}

*\% MV - the percentage morphologically verified; \%DCO - the percentage reported from death-certificate evidence only

Table 3. Duplicative Data for All New Invasive Cancer Cases, All Registered Cases; Bangkok Cancer Registry, 2013-2015*

\begin{tabular}{lcc}
\hline Place of registration & Number & $\%$ \\
\hline Bangkok only & 43,074 & 96.1 \\
Duplicated in other regions & 1,739 & 3.9 \\
North & 165 & \\
North East & 174 & \\
Central & 1,126 & \\
East & 201 & \\
South & 73 & \\
Total & 44,813 & 100 \\
\hline
\end{tabular}

(3.7\% compared with $6.0 \%$ ) and the \% morphologically verified (MV) (81.7\% compared with $75.9 \%$ ) (Table 2 ). Exceptions applied, however, with a higher $\% \mathrm{DCO}$ for liver and lung cancer in females and a lower \%MV for colorectal cancer and breast in females. No difference in $\% \mathrm{MV}$ was apparent by sex for cancers of the lung.

Most cases $(n=36,327)$ were found from official records to be Bangkok residents $(81.1 \%)$, the majority $(87.0 \%)$ being longstanding residents of 10 years or more. Of the 8,486 found to be residents of other provinces of Thailand, the majority $(81.3 \%)$ were from the Central province, $7.3 \%$ from the East, $5.4 \%$ from the North East, $3.1 \%$ from the North and $2.9 \%$ from the South. A past residency history in Bangkok applied to 3,941 (46.4\%) of the registered cases found to be current residents of these other provinces.

In general, most cases $(40,480,90.3 \%)$ were diagnosed and had their treatment completed at the one hospital and $4,333(9.7 \%)$ received care at two or more hospitals. Most cases $(43,074,96.1 \%)$ were recorded only on the Registry, but $1,739(3.9 \%)$ had duplicative registrationsin another region, 1,126 of them $(64.7 \%)$ in the Central province (Table 3).

\section{Cases registered among Bangkok residents}

The percentage of cases registered on the Registry with an official Bangkok residential address was $81.1 \%$, i.e., $18.9 \%$ fewer than for all cases. The corresponding percentage was $19.7 \%$ lower for males and $18.3 \%$ lower for females. As a result, age-standardized incidence rates for all cancers combined among Bangkok residents were $20.0 \%$ lower in males and $18.8 \%$ lower in females after subtracting non-residents from the total (Table 1). The effect of excluding non-residents varied by primary cancer site, for example: in males, from $14.8 \%$ for lung to $25.9 \%$ for colorectal cancer; and in females, from $12.9 \%$ for lung to $24.0 \%$ for cervical cancer.

\section{Discussion}

Data quality indices for the Registry generally fall 
within the ranges seen for other Asian populations. The percentages of cases morphologically verified for all sites combined in 2013-2015 are similar: in males, to those reported for Beijing, Osaka and Busan; and in females, to those reported for Hong Kong, Mumbai and Hiroshima (Forman et al., 2014). Similarly, the percentages registered on the basis of death certificate evidence only fell within the range for other Asian populations, being similar for all sites combined: in males, to those reported for Nagasaki and Mumbai; and in females, to those reported for Manilla and Fukui (Forman et al., 2014).

Data quality issues have presented, as experienced in many large cities, due to difficulties aligning numbers of cancers registered with the source population (in this study, the officially verified population of Bangkok). Our results indicate that of the cancer cases registered by the Registry in 2013-2015, 81.1\% had an official residential address in Bangkok. There are a number of possible reasons for the registration of non-residents, including the inadvertent inclusion of: (1) patients from other provinces attending Bangkok temporarily for clinical care; (2) patients from other provinces working in Bangkok and using Bangkok clinical services; and (3) former residents of Bangkok still reporting a Bangkok residency after purchasing a new house outside Bangkok (note: these cases probably contributed to the 3,941 cases found in this study to have previously lived Bangkok).

Bangkok is a relatively expensive city. While many people choose to work there because of employment opportunities, many have the intention of moving to another region after retirement. In addition, some Bangkok residents purchase and move to new housesthat are more spacious outside Bangkok, due to price advantages, but their addresses on the Registry may remain unchanged. The population is therefore quite mobile and difficult to define from health records. This problem has been overcome in the present studythrough the use of official residential addresses from the Ministry.

The Ministry of Public Health now encourages all hospitals to use an ID smart card reader to record addresses, reduce typing errors, and save time completing hospital record cards. If this can be fully implemented throughout the country, patients will have only one address and duplications will be avoided.Periodic checks against National Civil Registration records of the Ministry of Interior, as undertaken in this study, can serve as an additional check and enable the completion of missing fields.

In addition to these data-quality gains, the use of official places of residence from the Ministry of Interior has greatly improved the quality of theRegistry reports by avoiding artificial inflation of incidence rates through inadvertent incorporation of cancer data for non-residents. It has also enabled avoidance of duplicative registration across multiple registries.

In conclusion, 1 . The differences in calculated incidence found in this study from using official address information are considered to be sufficient in magnitude to justify the routine use ofofficial residential data for calculating cancer incidence rates.
2. It is likely that if cancer rates are to be compared with those of other Asian cities as benchmarks, equivalent methodologies would be needed for all cities that, like Bangkok, are heavily used by residents from other areas.

3. Accurately defining cancer incidence is fundamentally important for evidence-based service planning and evaluating the impact of preventive initiatives across the Thai population.

\section{Funding}

National Cancer Institute, Department of Medical Services, Ministry of Public Health.

\section{Conflict of interest Nil.}

\section{Acknowledgements}

The authors gratefully acknowledge all public and private hospitals, university hospitals, and pathology laboratories that have provided these clinical data. We would like express our gratitude to the devoted staffs of cancer registry unit of our institute.

\section{References}

Forman D, Bray F, Brewster DH, et al (2014). Cancer Incidence in Five ContinentsVol.X. IARC Scientific Publications No. 164.Lyon, France: International Agency for Research on Cancer.

Imsamran W, Chaiwerawattana A, Wiangnon S, et al (2015). Cancer in Thailand, Vol VIII, 2010-2012. Bangkok; New Thammada Press (Thailand) Co., Ltd., Thailand.

MacLennan R (1991). Items of patient information which may be collected by registries. In 'Cancer Registration: Principles and Methods', Jensen OM, Parkin DM, MacLennan R, Muir CS and Skeet RG, (eds.), 43-46, IARC Scientific Publications No. 95, Lyon, International Agency for Research on Cancer.

Martin N, Sontipong S, Chindavijak K, et al (2001). Cancer Incidence in Bangkok 1993-1997. National Agricultural Extension and training Canter Printing house. Nakhon Pathom, Thailand.

National Cancer Institute, Thailand (NCI) (2017). Thai Cancer based report online [Internet]. Bangkok: NCI, Thailand. http://tcb.nci.go.th/CWEB/cwebBase. do?mode=initialApplication.Accessed 10 May 2018.

National Health Security Office (NHSO) (2012). Thailand's Universal Coverage Scheme: Achievements and Challenges. An independent assessment of the first 10 years (2001-2010). Nonthaburi, Thailand: Health Insurance System Research Office.

NationalIdentity Card Act. B.E.2505 (1962). Royal Thai Government Gazette, No.79, pp 22, Section number 115.

Office of the National Economic and Social Development Board (NESDB) (2017). Population projection for each province in Thailand, 2010-2030[Internet] http://social.nesdb.go.th/ SocialStat/StatReport_Final.aspx?reportid=1256\&template $=2 \mathrm{R} 1 \mathrm{C} \&$ yeartype $=\mathrm{M} \&$ subcatid $=1$.Accessed 10 May 2018 .

Tangcharoensathien V, Witthayapipopsakul W, Panichkriangkrai W, Patcharanarumol W, Mills A (2018). Health systems development in Thailand: a solid platform for successful implementation of universal health coverage. Lancet, 391, 1205-23. 
Vatanasapt V, Martin N, Sriplung H, et al (1993). Cancer in Thailand 1988-1991. IARC Technical Report no.16. Lyon, France: International Agency for Research on Cancer.

WHO (2000). International Classification of Diseases for Oncology. Geneva: World Health Organization.

\section{(ब) $(1)$}

This work is licensed under a Creative Commons AttributionNon Commercial 4.0 International License. 